\title{
High-quality phase-shifted Bragg grating sensor inscribed with only one laser pulse in a polymer optical fiber
}

\author{
C. A. F. Marques ${ }^{1,2 *}$, A. Pospori ${ }^{3}$, L. Pereira 2 , S. Marques ${ }^{4}$, P. Antunes ${ }^{2}$, O. Bang ${ }^{5}$, D. J. Webb ${ }^{3}$, and P. André
}

\author{
${ }^{1}$ Instituto de Telecomunicações, Campus de Santiago, 3810-193 Aveiro, Portugal \\ ${ }^{2}$ University of Aveiro, Physics Department \& I3N, Campus de Santiago, 3810-193 Aveiro, Portugal \\ ${ }^{3}$ Aston Institute of Photonic Technologies, Aston University, Aston Triangle, B4 7ET Birmingham, UK \\ ${ }^{4}$ University of Science and Technology, Wybrzeze Wyspianskiego 27, 50-370 Wroclaw, Poland \\ ${ }^{5}$ Department of Photonics Engineering, Technical University of Denmark, DK-2800 Kgs. Lyngby, Denmark \\ ${ }^{6}$ Instituto de Telecomunicações and Department of Electrical and Computer Engineering, Instituto Superior Técnico, University of Lisbon, 1049-001 \\ Lisbon, Portugal \\ "cmarques@av.it.pt
}

\begin{abstract}
We present the first phase-shifted polymer optical fiber Bragg grating sensor inscribed with only one KrF laser pulse. The phase shift defect was created directly during the grating inscription process by placing a very narrow blocking aperture, in the center of the UV beam. One laser pulse with a duration of 15 ns and energy $6.3 \mathrm{~mJ}$ is adequate to introduce a refractive index change of $0.69 \times 10^{-4}$ in the fiber core. The high-quality produced Bragg grating structure rejects $16.3 \mathrm{~dB}$ transmitted power, thus providing $97.6 \%$ reflectivity, which is well suited for photonic applications. The transmission notch depth is about $10 \mathrm{~dB}$ and very sharp notches of $3 \mathrm{~dB}$ width ranging from $14 \mathrm{pm}$ is reported. The temperature, strain, and pressure response of the sensor has been characterized showing promising results in applications that require high-precision measurements. The ability to inscribe these high-quality sensors effectively can significantly reduce their production cost in industry.
\end{abstract}

Keywords - Optical sensors, polymer fibers, fiber Bragg gratings, phase-shift, annealing.

\section{INTRODUCTION}

As fabrication technology of FBGs in silica fibers is already well established, the Bragg grating-based phase-shifted inscribed in such fibers were studied for numerous practical applications due to their simplicity, small size and increased measurement resolution. In recent years, the technology of fabrication of FBGs in polymer optical fibers (POFBGs) has been intensively developed using uniform FBGs, tilted FBGs, chirped FBGs, or FBG-based Fabry-Perot interferometer $[1,2]$. Specific material properties, such as low Young's modulus (about $3 \mathrm{GPa}$ compared to $72 \mathrm{GPa}$ for glass), a wider range of strain that the POFs can withstand as well as biological compatibility open a variety of new applications unattainable for silica fiber [1-4].

In the last few years, there is an increase in the research of POF technology in the biomedical applications [5-8]. Recently, Broadway et al showed the scope and potential that POF offers for endoscopic implementation [6]. Also, the same group presented the first opto-acoustic measurements obtained using a mPOF uniform Bragg grating and presented the lateral directivity pattern of an ultrasound sensor over a frequency range of $1-50 \mathrm{MHz}[7,8]$. These works showed that the acoustic sensitivity for POF is thirteen times higher than for silica fiber. However, they discussed the impact of the use uniform FBGs to mitigate some problems encountered, where a potential transition from uniform FBGs to Fabry-Perot cavities or phaseshifted POFBGs (PS-POFBGs) could be a validated option. The goal with that is to deliver a narrower spectral profile, which it can potential used to achieve higher sensitivities. Until now, the literature only provides a single report on a PS-FBG in POFs with quality, which was for use at $\mathrm{THz}$ frequencies by using a point-by-point FBG fabrication method [9]. However, this latter method should be avoided due to long-time consumption and consequently the high cost setup used to produce these gratings at $850 \mathrm{~nm}$ or $1550 \mathrm{~nm}$ spectral regions.

For the first time, we report high-quality PS-POFBGs inscribed with only one krypton fluoride laser pulse at $850 \mathrm{~nm}$ region with high-quality. The device has been inscribed in a single-mode poly (methyl methacrylate) optical fiber, with a core doped with benzyl dimethyl ketal (BDK) for photosensitivity enhancement. The phase shift defect was created directly during the grating inscription process by placing a very narrow blocking aperture, in the center of the UV beam. The high-quality produced Bragg grating structure rejects 16.3 $\mathrm{dB}$ transmitted power, thus providing $97.6 \%$ reflectivity, which is well suited for sensing applications. The PS-POFBG's temperature, strain, and pressure characteristics are also experimentally studied. These PS-POFBGs can be useful optical devices not only for optical sensing but also for different applications such as in multiple wavelength fiber lasers or filters.

\section{EXPERIMENTAL SETUP}

A $\mathrm{KrF}$ excimer laser system operating at $248 \mathrm{~nm}$ wavelength has been used for the PS-POFBGs inscription. The laser pulse duration is $15 \mathrm{~ns}$ and the pulse energy can be pre-set; in this study, the energy more suitable for this grating type is $6.3 \mathrm{~mJ}$, where it was investigated with detail in our previous work [10]. Since the fiber core is doped with BDK for photosensitivity 
enhancement, where the absorption coefficient of BDK is much higher at wavelengths shorter than $325 \mathrm{~nm}$, shorter laser inscription wavelengths enhance its effects [10]. The fiber is 3ring microstructured with a hole diameter $1.74 \mu \mathrm{m}$ and average pitch $3.7 \mu \mathrm{m}$. Its core size is $8 \mu \mathrm{m}$ and its external diameter 130 $\mu \mathrm{m}$. A cross-section image of the POF used in this work is shown in Fig. 1 (a). The laser beam is focused in the fiber core utilizing a plano-convex cylindrical lens with effective focal length of $200 \mathrm{~mm}$. The effective spot size of the beam on the fiber surface is $20 \mathrm{~mm}$ in width and $32.4 \mu \mathrm{m}$ in height. For the PS-POFBG inscription, we used the typical uniform phase mask technique with $10 \mathrm{~mm}$ in width with a period of $\Lambda_{\mathrm{PM}}=567.8 \mathrm{~nm}$ and it can produce gratings with Bragg wavelengths approximately at $\lambda_{\mathrm{B}}=844 \mathrm{~nm}$.

Before the inscription, POF pieces of length between $15 \mathrm{~cm}$ and $35 \mathrm{~cm}$ were glued into demountable $\mathrm{FC} / \mathrm{PC}$ connectors to facilitate the POFBG interrogation. The average loss per connector is less than $1 \mathrm{~dB}$. Different fiber pieces were preannealed with the same procedure used in [10], where the fiber was placed for 15 minutes in a container filled with water that was heated at $60 \pm 1{ }^{\circ} \mathrm{C}$ process and also to remove possible twists present on it. Also, considering our recent results [10], we can conclude that a single laser pulse in a pre-annealed POF is adequate for a POFBG inscription.

The phase shift defect was created directly during the grating inscription process by placing on the phase mask a very narrow blocking aperture (a metal wire with $40 \mu \mathrm{m}$ diameter) in the center of the UV beam, as it is schematically illustrated in Fig. 1 (b). The phase shift occurs because, where the UV beam is blocked, the mean fibre index is less than in the rest of the grating. It resulted in the inscription of two FBGs separated by a very small gap in a single fabrication process, producing a phase shift on the structure. To observe the grating spectrum in transmission, the POF is connected between the super luminescent diode and the optical spectrum analyzer.

\section{RESULTS}

Fig. 1 (c) gives the transmission and reflection spectra of inscribed PS-FBG after an UV pulse. We can see that there are two main dips in each transmission spectrum because the PSPOFBG is successfully inscribed. The refractive index modulation produced by narrow wire acts as a phase shift of the grating during the inscription process. The transmission losses of the 1 st and 2 nd dips are about $-16.25 \mathrm{~dB}$ and $-13.17 \mathrm{~dB}$, and the central wavelengths are $844.126 \mathrm{~nm}$ and $844.187 \mathrm{~nm}$, respectively. In this case, the channel space of these two dips is about $61 \mathrm{pm}$, and the transmission notch depth is about $10 \mathrm{~dB}$. We can fabricate the PS-POFBGs in transmission with very sharp notches of $3 \mathrm{~dB}$ width $(\sim 14 \mathrm{pm})$ as shown in the inset of Fig. 1 (c) depending the total grating length (in this case with 10 mm length). Fig. 1 (c) also shows a comparison of simulated and experimental results showing a good agreement between them. The simulation of the PS-POFBG spectrum can be accomplished using the transfer matrix method [12]. The success rate of the PS-POFBG photo-inscription is quite high more than $98 \%$ - give us a high repeatability of fabrication. Comparing these PS-POFBG spectra with uniform POFBG ones, the slopes of the PS-POFBG compared with normal
POFBG in the linear regions are several orders enhanced, which will result in the same input signal yielding detected signals with amplitudes that differ by a higher factor [11]. Though the dynamic range is smaller in the PS-POFBG because of the narrow peak, for the application of ultrasonic detection, this is not a problem since the strain is always small.

(a)
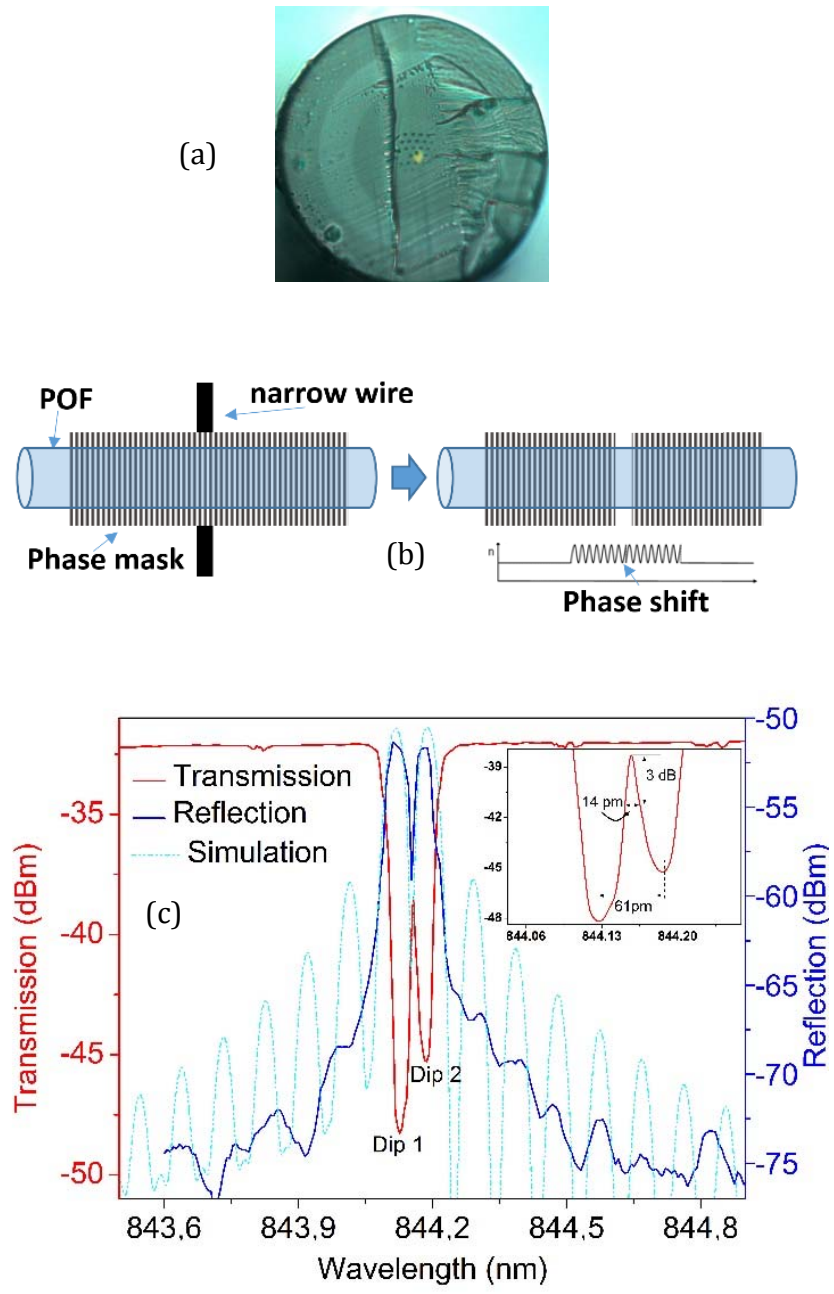

Figure 1. (a) Fiber cross-section. (b) A schematic configuration of the PSPOFBG fabricated with a very narrow metal wire positioned in the center of the UV beam. (c) Transmission and reflection/simulation spectra of inscribed PS-POFBG after an UV pulse UV beam.

After the successful PS-POFBGs inscription a characterization to temperature, strain and pressure was done, in order to show the spectral dependence of the Bragg peak under different external conditions.

The results for the temperature, strain and pressure are shown in Figs. 2 (a)-(c), respectively. The change in Bragg wavelength over the $30^{\circ} \mathrm{C}$ temperature variation was obtained giving $-1.71 \mathrm{~nm}$. The obtained temperature sensitivity was $57.0 \pm 4.1 \mathrm{pm} /{ }^{\circ} \mathrm{C}$, after fitting to a linear model (see Fig. 2(a)). Fig. 2(a) shows that $1.71 \mathrm{~nm}$ tuning range can be achieved only with the temperature variation of $30^{\circ} \mathrm{C}$, which is larger than the achieved in silica PS-FBG by the several hundred-degree temperature variation. Fig. (b) displays the strain response of the 
PS-POFBG shows a good linearity and repeatability, and the strain sensitivity is $0.76 \pm 0.01 \mathrm{pm} / \mu \varepsilon$, which is a little larger than $0.7 \mathrm{pm} / \mu \varepsilon$ of the silica PS-FBG reported in the literature [13]. From Fig. 2 (c), we can notice that the central wavelengths shift linearly with increasing and decreasing the pressure for two dips at a pressure sensitivity of $0.46 \pm 0.03 \mathrm{pm} / \mathrm{kPa}$, which is more than 2 times sensitive compared with uniform POFBGs [14].
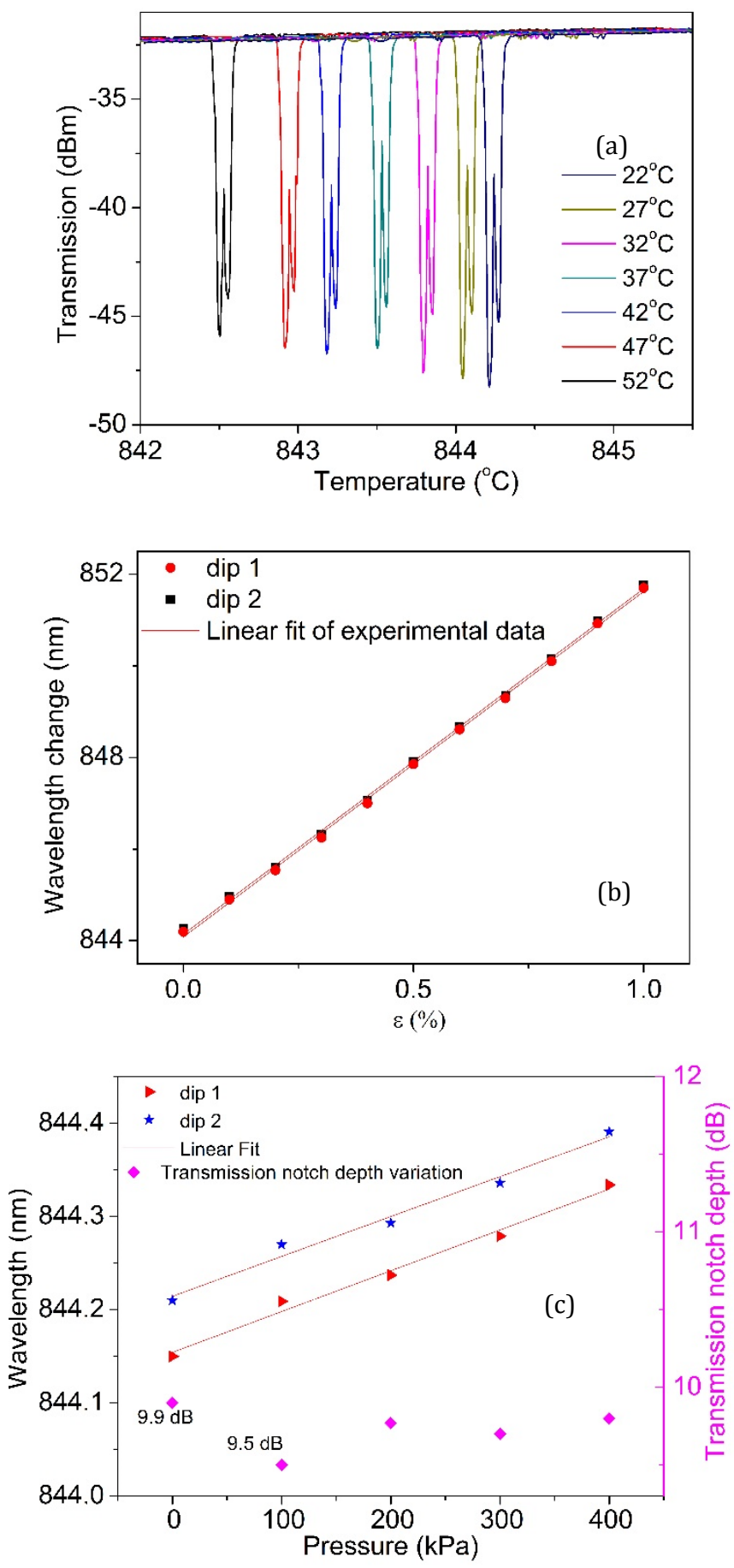

Figure 2. Characterization of a PS-POFBGs for: temperature (a), strain (b) and pressure $(\mathrm{c})$.

\section{CONCLUSIONS}

A fast inscription with only one $\mathrm{KrF}$ laser pulse of highquality phase-shifted Bragg gratings in a doped PMMA mPOF is reported for the first time. The gratings were created through the phase mask technique, using $248 \mathrm{~nm}$ UV light. The phase shift defect was created directly during the grating inscription process by placing a very narrow blocking aperture, in the center of the UV beam. The PS-POFBGs were created at $850 \mathrm{~nm}$ region and produced Bragg grating structure rejects $16.25 \mathrm{~dB}$ transmitted power, which is well suited for photonic applications. The spectral dependence of the PS-POFBG with temperature, strain and pressure were analyzed. The optical characteristics of the achieved PS-POFBGs show promising results in applications that require high-precision measurements such as ultrasonic detection. The optimization of the FBG response by changing the narrow wire diameter or placing multiple narrow wires in different zones of the phase mask are in progress.

\section{ACKNOWLEDGMENT}

This work was supported by Fundação para a Ciência e Tecnologia (FCT)/MEC through national funds and when applicable co-funded by FEDER - PT2020 partnership agreement under the project UID/EEA/50008/2013. C.A.F. Marques also acknowledges the financial support from FCT through the fellowship SFRH/BPD/109458/2015. The research leading to these results has also received funding from the Marie Curie Actions of the European Union's 7th Framework Programme FP7/2007-2013/ under REA grant agreement No. 608382. We are grateful to Dr. David Sáez-Rodríguez for providing the polymer optical fiber used in this work.

\section{REFERENCES}

[1] C. A. F. Marques, D.J. Webb, P. Andre, "Polymer optical fiber sensors in human life safety", Optical Fiber Technology 36, 144 (2017).

[2] D. J. Webb, "Fibre Bragg grating sensors in polymer optical fibres", Meas. Sci. Technol. 26, 092004 (2015).

[3] C.A.F. Marques, P. Antunes, P. Mergo, D. J. Webb, P. André, "Chirped Bragg gratings in PMMA step-index polymer optical fiber," IEEE Photonics Technol. Lett. 29, 500 (2017).

[4] C. A. F. Marques, G. D. Peng and D. J. Webb, "Highly sensitive liquid level monitoring system utilizing polymer fiber Bragg gratings," Opt. Express 23, 6058 (2015).

[5] D. Gallego, and H. Lamela, "High-sensitivity ultrasound interferometric single-mode polymer optical fiber sensors for biomedical applications," Opt. Lett., 34, 1807 (2009).

[6] C. Broadway, D. Gallego, G. Woyessa, A. Pospori, G. Carpintero, O. Bang, K. Sugden, H. Lamela, "Fabry-Perot micro-structured polymer optical fibre sensors for opto-acoustic endoscopy," Proc. SPIE. 9531, Biophotonics South America, 953116 (2015).

[7] C. Broadway, D. Gallego, A. Pospori, M. Zubel, D. J. Webb, K. Sugden, G. Carpintero, H. Lamela, "A compact polymer optical fibre ultrasound detector”, Proc. SPIE. 9708, Photons Plus Ultrasound: Imaging and Sensing, 970813 (2016).

[8] D. Gallego, H. Lamela, "Microstructured polymer optical fiber sensors for optoacoustic endoscopy,". Proc. SPIE 10064, Photons Plus Ultrasound: Imaging and Sensing, 1006412 (2017).

[9] S. F. Zhou, L. Reekie, Y. T. Chow, H. P. Chan, and K.i M. Luk, "PhaseShifted Fiber Bragg Gratings for Terahertz Range", IEEE Photon. Technol. Lett. 24, 1875, (2012). 
[10] A. Pospori, C. A. F. Marques, D. J. Webb, P. André "Polymer optical fiber Bragg grating inscription with a single UV laser pulse", Opt. Express 25, 9028 (2017).

[11] Q. Wu and Y. Okabe, "High-sensitivity ultrasonic phase-shifted fiber Bragg grating balanced sensing system," Opt. Express 20, 28353 (2012).

[12] T. Erdogan, "Fiber grating spectrum", IEEE/OSA Journ. of Lightw. Techn. 15, 1277 (1997).
[13] S. F. O. Silva, L. A. Ferreira, F. M. Araújo, J. L. Santos, O. Frazão, "Fiber Bragg grating structures with fused tapers," Fiber Integr. Opt. 30, 9 (2011).

[14] K. Bhowmik et al., "Experimental Study and Analysis of Hydrostatic Pressure Sensitivity of Polymer Fibre Bragg Gratings," IEEE/OSA Journ. of Lightw. Techn. 33, 2456 (2015). 